\title{
Alívio da dor em neonatos prematuros durante a aspiração de vias aéreas superiores comparando posicionamentos
}

\author{
Pain relief in premature neonates during \\ upper airway aspiration comparing positions
}

\section{Marimar Goretti Andreazza1 \\ Arlete Ana Motter ${ }^{2}$ (1) \\ Monica Lima $\mathrm{Cat}^{3}$ (D) \\ Regina Paula Guimarães Vieira Cavalcante da Silva ${ }^{4}$}

1-3Universidade Federal do Paraná (Curitiba). Paraná, Brasil. marimar.andreazza@hc.ufpr.br, arlete.motter@uol.com.br, monica.lima.ufpr@gmail.com ${ }^{4}$ Autora para correspondência. Universidade Federal do Paraná (Curitiba). Paraná, Brasil. reginavcs@uol.com.br

RESUMO | INTRODUÇÃO: Os recém-nascidos prematuros frequentemente passam por longos períodos internados em unidades de terapia intensiva neonatal. Nesses pacientes, a dor, resultado das intervenções intrusivas é motivo de preocupação das equipes multidisciplinares. OBJETIVO: Identificar a melhor intervenção para alívio da dor durante a aspiração de vias aéreas superiores, comparando posicionamento no ninho com contenção em cueiro. MÉTODO: Foi realizado um estudo do tipo Antes e Depois em recém-nascidos prematuros com menos de 32 semanas de idade gestacional ao nascimento e que necessitassem de aspiração de vias aéreas na primeira semana de vida. No Grupo Antes, os pacientes foram posicionados no ninho, e no Grupo Depois, permaneciam contidos com cueiro. $O$ desenho do estudo permitiu que os pacientes fossem controles deles mesmos e participassem do estudo mais de uma vez, conforme a necessidade de aspiração. O estudo teve aprovação do Comitê de Ética em Pesquisa com Seres Humanos. RESULTADOS: Participaram 22 recém-nascidos prematuros que foram submetidos a 100 procedimentos de aspiração do trato respiratório. Conforme análise estatística dependente constatou-se que os indivíduos que haviam pontuado dor no momento em que estavam posicionados no ninho, passaram a não pontuar quando contidos, pela Neonatal Infant Pain Scale $(p=0,001)$ e Premature Infant Pain Profile $(p=0,01)$. Observou-se uma menor variação da frequência cardíaca no grupo contenção. CONCLUSÃo: A dor está presente na aspiração de secreções das vias aéreas. A frequência cardíaca variou percentualmente menos no grupo contenção. Os pacientes posicionados em contenção passaram a não pontuar escore positivo para dor.

PALAVRAS-CHAVE: Dor. Contenção facilitada. Neonatologia. Unidades de Terapia Intensiva Neonatal.
ABSTRACT | INTRODUCTION: Premature newborns have survived long periods of time in neonatal intensive care units. In these patients, the pain, the result of intrusive interventions, is a concern of multidisciplinary teams. OBJECTIVE: To identify the best intervention for pain relief during the suction of the upper airway, nest positioning with containment in swaddling. METHODS: The Before and After type of study was conducted in preterm infants with less than 32 weeks at birth, who required aspiration of the airways in the first week of life. In the Before Group, patients were placed in the nest, and in the After Group, they remained contained in swaddling. The design of the studio allows patients to have their own controls and participate in the study more than once, according to the need for suction. The study was approved by the Human Research Ethics Committee. RESULTS: 22 premature newborns participated who were submitted to 100 procedures for suction of the upper airway. According to the dependent statistical analysis, it will be found that the individuals who usually score the pain at the time they place themselves in the score, when they are contained, by the Neonatal Pain Scale $(p=0.001)$ and the Profile of Premature Infant Pain $(p=0.01)$. Less variation in heart rate was observed in patients in the containment group. CONCLUSION: The pain is present in the suction of the upper airway. Heart rate varies in a smaller percentage in the containment group. The patients of containment did not obtain a positive pain score.

KEYWORDS: Pain. Facilitated tucking. Neonatology. Neonatal Intensive Care Units. 


\section{Introdução}

A grande maioria dos recém-nascidos (RN) internados em uma unidade de terapia intensiva neonatal (UTIN) pulmonar, necessitam de fisioterapia respiratória e aspiração de vias aéreas. Estes procedimentos podem ser causa importante de dor e alterações nos parâmetros fisiológicos ${ }^{1}$. A aspiração de vias aéreas é o procedimento doloroso realizado com maior frequência, sendo responsável por $23 \%$ dos realizados com RN nas UTIN².

Contudo, apesar do grande número de procedimentos invasivos realizado nessas unidades existe uma contradição entre o uso de analgesia e a frequência de procedimentos dolorosos 3 . Várias terapias não farmacológicas têm sido propostas para controle e modulação da dor e do desconforto nesta população, com efeitos comprovados, apesar dos mecanismos fisiológicos não estarem completamente esclarecidos 4 .

O reconhecimento da dor no recém-nascido prematuro (RNPT) ainda representa um desafio, mesmo sendo expostos com frequência a procedimentos dolorosos. A dificuldade de avaliação da dor nesta população consiste no fato de que estes a expressam de forma sutil, sendo esta reação também dependente, entre outros fatores, da idade gestacional, da sensibilidade do instrumento utilizado, do conhecimento para interpretar as ferramentas de avaliação e da percepção da dor que o cuidador possui.

Desse modo, em pacientes não verbais, como os RNPT, existe a necessidade de se utilizarem instrumentos para avaliar a dor em tempo real e com precisão, a fim de protegê-los dos danos e propor uma forma de alívio ${ }^{5}$. Este fato é motivo de preocupação das equipes assistenciais multidisciplinares, pois, além das questões éticas, sabe-se que a dor sensibiliza as vias neurais imaturas, e pode trazer, em longo prazo, repercussões neurológicas, tais como alterações de comportamento, de atenção e aprendizagem ${ }^{6}$.

Diante desse contexto, compreendendo que a prevenção e o tratamento da dor são direitos fundamentais, independente da compreensão e maturação neurológica, emerge a preocupação do profissional fisioterapeuta na UTIN em proporcionar ao RNPT cuidado de qualidade, visando além da desobstrução brônquica e da permeabilidade das vias aéreas, o conforto, por meio de medidas não farmacológicas. O estudo tem relevância uma vez que o próprio
Ministério da Saúde preconiza medidas de atenção humanizadas ao RNPT deste modo, o objetivo do estudo foi identificar a melhor intervenção para alívio da dor durante a aspiração de vias aéreas superiores, comparando posicionamento no ninho com contenção em cueiro.

\section{Método}

O projeto de pesquisa foi submetido à avaliação pelo Comitê de Ética em Pesquisa em Seres Humanos da instituição, e foi aprovado sob o número 706.623 (CAAE 19182613.5.0000.0096). Utilizou-se o Termo de Consentimento Livre e Esclarecido (TCLE), obrigatório para pesquisas em seres humanos, (Resolução $n^{\circ} 466$ de 12/12/2012), o qual foi assinado pelos pais.

O estudo foi do tipo Antes e Depois, realizado na Unidade de Terapia Intensiva Neonatal de um hospital público universitário, no período entre março de 2014 a maio de 2015.

A coleta de dados foi realizada na primeira semana de vida e fizeram parte do estudo os recém-nascidos que obedeceram aos seguintes critérios de inclusão: idade gestacional entre 24 a 32 semanas avaliada pela ultrassonografia fetal precoce, com idade cronológica entre o segundo e o sétimo dia de vida, ter presença de secreção na ausculta pulmonar avaliada pelo médico indicando necessidade de fisioterapia respiratória e aspiração de vias aéreas superiores, por duas vezes ao dia no mínimo, independente de estarem em ar ambiente ou sob suporte ventilatório invasivo ou não invasivo. Foram considerados como critérios de exclusão: Presença de quaisquer síndromes genéticas e malformações congênitas de cabeça, pescoço ou sistema nervoso central; pacientes criticamente doentes, Snappe II maior que 70, transferência ou óbito antes da primeira coleta de dados, nascidos em períodos de pausa da pesquisa, como férias da pesquisadora, feriados prolongados.

Foi utilizada amostragem de conveniência e considerando os critérios de inclusão e exclusão, constituiuse a população de estudo 22 recém-nascidos prematuros, sendo que o desenho do estudo determinou que os pacientes fossem o controle deles mesmos, e participassem do estudo diversas vezes durante a primeira semana de vida, conforme suas necessidades clínicas de fisioterapia e aspiração de secreções. 
Todos os participantes receberam os procedimentos de manhã e à tarde, após um período de pelo menos 1 hora e 30 minutos sem receber outras intervenções, como troca de fraldas, coleta de sangue, manuseios da equipe. Totalizando 100 procedimentos de fisioterapia seguidos de aspiração. Os RNPT que participaram do estudo receberam atendimento de fisioterapia respiratória e aspiração do trato respiratório com adoção de medidas posturais, e foram divididos em dois grupos: Antes, denominado Ninho e Depois, Contenção.

Durante o procedimento de fisioterapia respiratória e aspiração do trato respiratório, no período da manhã, os recém-nascidos foram posicionados no ninho, grupo Antes ou Ninho. Permaneciam em supino dentro do ninho, em semiflexão de membros inferiores, dentro da incubadora, conforme padrão de posicionamento utilizado na unidade. O ninho é confeccionado por cueiros dobrados e colocados ao redor do recém-nascido.

No segundo atendimento, no período da tarde, foram posicionados no ninho e também com contenção por meio de cueiros, grupo Depois, ou Contenção. A contenção restringe a movimentação do recém-nascido, mas não impede que o mesmo se movimente espontaneamente, sendo perceptível ao examinador a presença dos movimentos de membros superiores e inferiores. Os recém-nascidos foram contidos mantendo o tórax descoberto para que fosse possível realizar a verificação da frequência respiratória. (FIGURA1)

Figura 1. Grupo antes e grupo depois
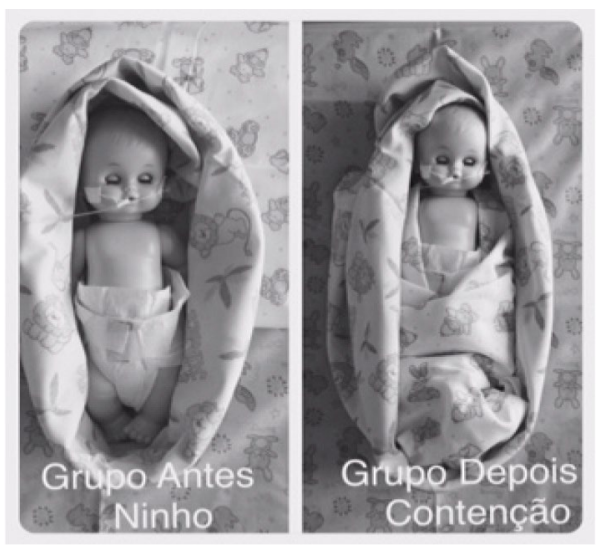

As respostas de dor foram avaliadas pelas escalas Neonatal Infant Pain Scale (NIPS) ${ }^{7}$ e Premature Infant Pain Profile (PIPP) ${ }^{8}$. O avaliador das escalas de dor foi o membro da equipe de enfermagem responsável pelo recém-nascido no momento da coleta, ou na impossibilidade deste, foi o membro da equipe de fisioterapia. Para realização deste estudo a equipe de enfermagem e fisioterapia atuantes no período da coleta foi treinada pela pesquisadora, sendo apresentados aspectos da manifestação da dor em recém-nascidos prematuros, como reconhecer a dor e como avalia-la, e as escalas NIPS e PIPP?

Foram coletados dados do prontuário como sexo, peso, idade gestacional, escore Snappe II (Score for Neonatal Acute Physiology with Perinatal Extension-II/ $)^{10}$ e escore Apgar. Registrou-se os parâmetros fisiológicos, frequência respiratória, frequência cardíaca, saturação periférica de oxigênio pela oximetria de pulso e avaliação da dor pelos escores NIPS e PIPP. Esses parâmetros foram anotados em três momentos, a saber: ao final da fisioterapia e imediatamente antes do procedimento de aspiração (T1), imediatamente após o término do procedimento (T2) e cinco minutos após o término do procedimento (T3). Nos últimos 5 minutos, entre os momentos T2 e T3, o paciente não era tocado por nenhum profissional.

Considerando a hipótese relacional, as variáveis de intervenção Ninho ou Contenção foram posicionadas como variáveis independentes e como variáveis dependentes o escore NIPS e PIPP. Todos os dados foram registrados no instrumento de coleta, digitados em planilha Excel, conferidos e exportados para o programa Statistic Statsoft ${ }^{\circledR}$. Inicialmente realizouse a estatística descritiva, com medidas de tendência central e de dispersão para as variáveis contínuas e frequência absoluta e relativa para as variáveis categóricas. A estatística inferencial foi realizada de acordo com os grupos de estudo e objetivos da pesquisa. Para análise dependente das variáveis categóricas foi realizado o teste Mc Nemar e para análises independentes foi realizado o teste Exato de Fisher. Para análise das variáveis contínuas de amostras dependentes foi realizado o Teste $T$ Student para amostras dependentes. Para todos os testes foi considerado o nível de significância de 5\%. 


\section{Resultados}

No período de coleta de dados nasceram 76 recémnascidos prematuros com menos de 32 semanas de idade gestacional. Dos quais 43 foram excluídos, 20 por não ter indicação para receber dois atendimentos diários de fisioterapia, 8 por transferência antes da primeira coleta de dados, 7 por óbito nas primeiras horas de vida, 4 por se apresentarem criticamente doentes, 3 nascidos malformados, e 1 por não concordar em participar. Sendo que, dos 33 que atenderam aos critérios de inclusão, 11 nasceram em períodos de pausa da pesquisa.

Os 22 recém-nascidos prematuros participantes do estudo tinham média de idade gestacional de 28,4 $( \pm 2,34)$ semanas e mediana de peso ao nascimento de 1025,45 gramas (variando de 510,0 - 2230,0 gramas), estando assim distribuídos quanto à categoria de peso: 50\% (11 pacientes) entre 500 e 1000 gramas, 27,3\% (6 pacientes) entre 1001 e 1500 gramas e $22,7 \%$ ( 5 pacientes) acima de 1501 gramas. Doze pacientes (55\%) eram do sexo masculino enquanto 10 (45\%) do sexo feminino. A maior parte $(75 \%)$ dos recém-nascidos apresentou escore de Apgar no quinto minuto maior ou igual a sete pontos, e apresentaram escore de SNAPPE-II com mediana de 23,2 variando de 5 a 62 pontos, mostrando assim que casos onde 0 escore era maior não participaram da pesquisa pois indicavam maior gravidade.

Os procedimentos de fisioterapia e aspiração do trato respiratório passavam previamente por avaliação clínica com ausculta pelo médico e pelo fisioterapeuta pesquisador, e havendo presença de secreção os pacientes participavam do estudo. A mediana de dias de vida no momento da coleta foi de 4 dias variando de 2 a 7 , sendo que 10 recém-nascidos tinham 2 dias de vida. Entre estes, 5 tinham peso menor que $1500 \mathrm{~g}$.

Dos 100 procedimentos coletados, 4\% estavam em ar ambiente, e $96 \%$ recebiam oxigênio suplementar sendo assim ofertado: cateter $12 \%$, continuous positive airway pressure (CPAP) 48\%, ventilação mecânica invasiva 36\%, não havendo diferença estatisticamente significativa entre os casos que pontuaram e não pontuaram dor pelas escalas NIPS e PIPP. $(p=0,54$ Teste exato de Fisher).

A primeira avalição dos escores de dor realizados no $\mathrm{T} 1$, imediatamente após o atendimento de fisioterapia, no grupo ninho houve somente uma vez que o recém-nascido pontuou dor e no grupo contenção em 3 procedimentos os pacientes pontuaram dor, pelos escores NIPS E PIPP. Pelo número insuficiente de casos com dor não foi possível realizar testes estatísticos.

A Tabela 1 apresenta a presença de dor nos momentos avaliados, T1, T2 e T3, pela escala NIPS e PIPP nos grupos Ninho e Contenção.

A comparação dos resultados entre grupos Ninho e Contenção, pela escala NIPS e PIPP de acordo com idade gestacional, no momento $\mathrm{T} 2$, imediatamente após a aspiração, está descrita na Tabela 2.

Na Tabela 3 foram detalhadas as áreas de concordância pelos escores NIPS e PIPP. Na avaliação pela NIPS os recém-nascidos que pontuaram dor no ninho, quando foram colocados em contenção dos 16 (32\%), $10(62,5 \%)$ passaram a não pontuar mais dor. Pelo escore PIPP, quando os indivíduos que pontuaram dor foram colocados em contenção, dos 26 (52\%), 10 $(38,5 \%)$ passaram a não pontuar mais dor.

Quanto a variação percentual dos dados fisiológicos entre os momentos $\mathrm{T} 1 / \mathrm{T} 2$, a frequência cardíaca no grupo Ninho variou 5,9 $(-7,9-81,9)$ e no grupo Contenção 1,3 $(-13,8-36,5)$, segundo o teste estatístico Mann-Whitney $(p<0,001)$. Os demais dados fisiológicos coletados foram frequência respiratória e saturação periférica de oxigênio não apresentaram diferença estatisticamente significativa, e estão demonstrados na Tabela 4. 
Tabela 1. Presença de dor pelo escore nips e pipp nos grupos ninho e contenção, nos momentos T1 - T2 - T3

\begin{tabular}{l|l|l|l}
\hline Momento & Ninho $(n=50)$ & Contenção $(n=50)$ & \\
\hline T1 & $1(2 \%)$ & $3(6 \%)$ & $*$ \\
T2 & $16(32 \%)$ & $11(22 \%)$ & $<0,001$ \\
T3 & $4(8 \%)$ & $2(4 \%)$ & $*$ \\
& & & \\
T1 & $1(2 \%)$ & $3(6 \%)$ & $*$ \\
T2 & $26(52 \%)$ & $22(44 \%)$ & 0,01 \\
T3 & $2(4 \%)$ & $0(0 \%)$ & * \\
\hline
\end{tabular}

NOTA: Teste estatístico: Teste Mc Nemar; *sem valor de $p$, devido número insuficiente de casos com dor.

Tabela 2. Presença de dor pós-aspiração pelo escore nips e pipp imediatamente após-aspiração, momento T2

\begin{tabular}{|c|c|c|c|c|c|c|c|c|}
\hline \multicolumn{5}{|c|}{ NINHO } & \multicolumn{4}{|c|}{ CONTENÇÃO } \\
\hline & & COM DOR & SEM DOR & $\mathrm{p}$ & COM DOR & SEM DOR & $\mathrm{p}$ & $\mathrm{p}^{*}$ \\
\hline \multirow[t]{3}{*}{$\Sigma \bumpeq$} & & $\begin{array}{c}\mathrm{n}=16 \\
32,0 \%\end{array}$ & $\begin{array}{c}n=34 \\
68,0 \%\end{array}$ & & $\begin{array}{c}n=11 \\
22,0 \%\end{array}$ & $\begin{array}{c}n=39 \\
78,0 \%\end{array}$ & & \\
\hline & IG(sem) & $29,1 \pm 2,3$ & $27,2 \pm 1,9$ & 0,008 & $29,4 \pm 1,9$ & $27,2 \pm 2,1$ & $<0,001$ & 0,72 \\
\hline & & $\begin{array}{c}\mathrm{n}=26 \\
52,0 \%\end{array}$ & $\begin{array}{c}n=24 \\
48,0 \%\end{array}$ & & $\begin{array}{c}n=22 \\
44,0 \%\end{array}$ & $\begin{array}{c}n=28 \\
56,0 \%\end{array}$ & & \\
\hline$\frac{1}{a}$ & IG(sem) & $27,6 \pm 2,4$ & $28,1 \pm 1,9$ & 0,46 & $27,4 \pm 2,6$ & $28,1 \pm 1,8$ & 0,28 & 0,78 \\
\hline
\end{tabular}

LEGENDA: $p^{*}$ entre grupos com dor: valor $p$ entre grupos que pontuaram dor; NIPS: Neonatal Infant Pain Scale; PIPP: Premature Infant Pain Profile; IG: idade gestacional; sem: semanas.

NOTA: Testes estatísticos: Teste T de Student para amostras dependentes

Tabela 3. Comparação das respostas no momento $T 2$

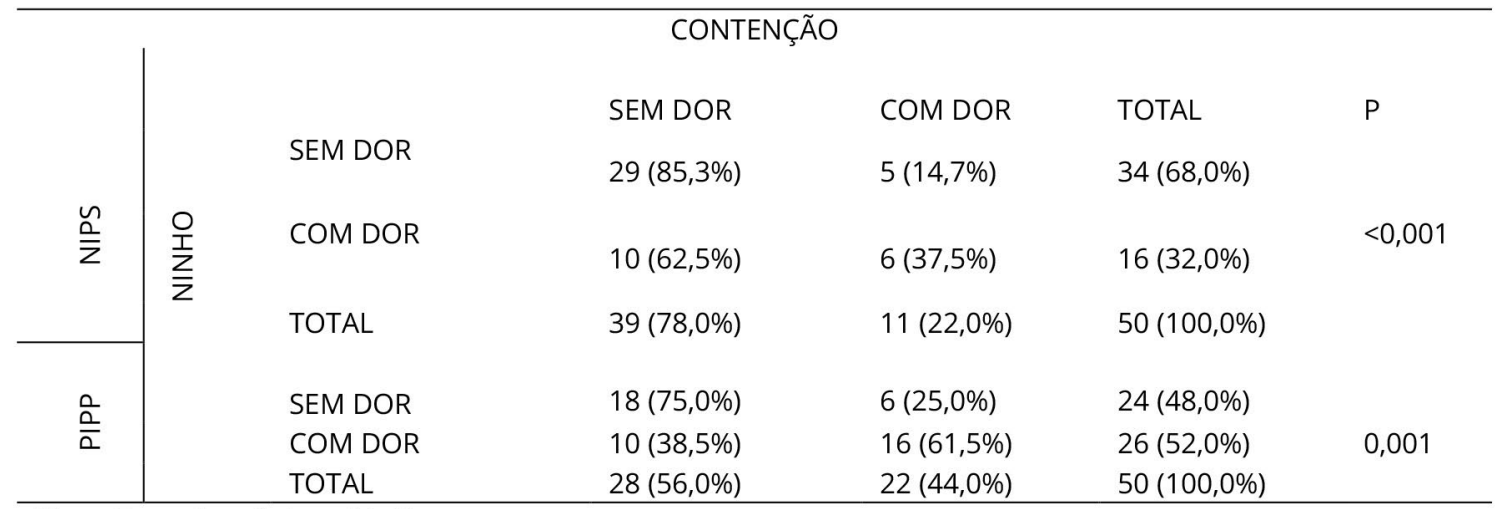

Nota: Teste Estatístico - Mc Nemar

Tabela 4. Variação percentual dos dados fisiológicos dos grupos ninho e contenção, entre os momentos T1/T2 e T2/T3.

\begin{tabular}{l|lll|lll}
\hline MOMENTOS & VARIÁVEIS & \multicolumn{2}{l|}{$\begin{array}{l}\text { GRUPO NINHO } \\
\text { MEDIANA (MIN-MAX) }\end{array}$} & $\begin{array}{l}\text { GRUPO CONTENÇÃO } \\
\text { MEDIANA (MIN-MAX) }\end{array}$ & $p$ \\
\hline \multirow{4}{*}{$\Delta \mathrm{T} 1 / \mathrm{T} 2$} & FC & 5,9 & $(-7,9-81,9)$ & 1,3 & $(-13,8-36,5)$ & 0,001 \\
& FR & 0 & $(-31,9137,9)$ & 1,82 & $(-34,5-87,5)$ & 0,83 \\
& SAT O 2 & $-2,0$ & $(-27,0-45,9)$ & $-2,0$ & $(-16,8-18,7)$ & 0,39 \\
$\Delta \mathrm{T} 2 / \mathrm{T} 3$ & FC & $-5,22$ & $(-32,6-8,7)$ & $-3,61$ & $(-20,9-23,6)$ & 0,31 \\
& FR & 0 & $(-39,6-56,4)$ & $-3,85$ & $(-30,0-60,0)$ & 0,58 \\
& Sat O & 3,1 & $(-4,2-38,6)$ & 2,1 & $(-6,1-20,2)$ & 0,54 \\
\hline
\end{tabular}

LEGENDA: $\triangle$ T1/T2: Variação em porcentagem entre o Tempo 1 e Tempo 2

$\triangle$ T2/T3: Variação em porcentagem entre o Tempo 2 e Tempo 3

FC: Frequência Cardíaca; FR: Frequência respiratória; Sat O2: Saturação periférica de oxigênio pela oximetria de pulso; Min: mínimo; Max: máximo

NOTA: Teste estatístico Mann-Whitney 


\section{Discussão}

O desenho do estudo propôs-se a avaliar a resposta diária da dor durante a aspiração de secreção das vias aéreas superiores, permitindo que o mesmo neonato participasse mais de uma vez, sendo observada a resposta de cada procedimento para análise estatística, e que os recém-nascidos fossem controles deles mesmos. Comparando-se a resposta do prematuro posicionado no ninho, durante a fisioterapia e aspiração de secreções com ele mesmo posicionado no ninho e com contenção por meio de cueiros. O procedimento de aspiração de secreções realizado por fisioterapeutas, dentre outros profissionais, foi eleito para ser estudado. Sendo importante ressaltar que foram realizados por necessidade do paciente, avaliados por ausculta pulmonar realizada pelo médico intensivista e pelo fisioterapeuta antes do atendimento e não para cumprir necessidades do estudo.

A necessidade clínica justificou o atendimento antes das 72 horas preconizadas na literatura para evitar as hemorragias peri-intraventriculares ${ }^{11}$. Quanto ao escore de gravidade Snappe II, pontuações variam de 0 a 162 onde valores mais altos indicam maior gravidade e maior o risco de mortalidade ${ }^{12}$, e os participantes do estudo obtiveram no máximo 62 pontos. Ressaltase que no presente estudo os recém-nascidos tinham menor média de idade gestacional comparando com outro estudo ${ }^{13}$. A literatura apresenta resultados de idades gestacionais diversas, e ligeiramente maiores, tornando as comparações difíceis.

Dentre os fatores contextuais que influenciam a resposta frente a estímulo doloroso, a idade gestacional é apontada como principal fator de influência, haja vista que a expressão facial tende a aumentar com a idade

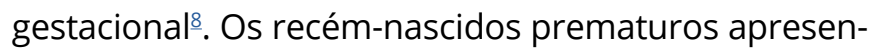
tam respostas mais sutis à dor, muitas vezes dificultando ao avaliador a percepção dessas respostas?

Os procedimentos intrusivos são dolorosos e o resultado dos escores de dor nos momentos T1, T2 e T3, apontaram a presença de dor no evento da aspiração de secreções, em ambos os grupos pesquisados, tendo diferença significativa entre os grupos Ninho e Contenção, quando avaliados pelo escores NIPS e PIPP. Corroborando com estudo que avaliou a presença de dor em procedimentos em uma UTIN como punção venosa, intubação e aspiração de vias aéreas ${ }^{14}$. Outro estudo que avaliou a aspiração de secreções com diferentes escalas também constatou a presença de dor intensa imediatamente após o procedimento $\frac{15}{}$.

Sabe-se que a dor está presente em muitos procedimentos durante o internamento nas UTIN e que o controle seu traz benefícios a longo prazo ${ }^{15}$. Devido ao caráter subjetivo da dor, para realizar a avaliação na população neonatal existem fatores que podem interferir na percepção e, sobretudo na observação das respostas apresentadas, entre estes estão a idade gestacional, peso de nascimento, e o avaliador ${ }^{16}$. A dor relacionada a procedimentos deve ser avaliada pela equipe multiprofissional, bem como a utilização de mais de uma escala, pois não existe um padrão ouro na avaliação. A equipe depois de treinada e familiarizada deve escolher entre as escalas validadas quais se adaptam ao perfil clínico dos pacientes ${ }^{17}$.

Quando se avalia a dificuldade da equipe em interpretar respostas apresentadas pelos RNPT, outro estudo concorda com os achados e afirma que neonatos com menos de 28 semanas expostos a estímulos nociceptivos apresentam respostas faciais menos intensas, quando comparados aos maiores de 32 semanas de idade gestacional. Estas diferenças fisiológicas foram explicadas pela maior imaturidade ao nascimento e ao número de procedimentos dolorosos vivenciados durante o internamento ${ }^{18}$.

Dentre as várias escalas de dor estão a NIPS, e a PIPP. A escala NIPS, que é composta por cinco indicadores comportamentais e um fisiológico, considera que o neonato apresenta dor quando se atribui quatro ou mais pontos 7.19 . A PIPP é composta por sete indicadores, a saber, idade gestacional, indicadores de sonovigília, expressão facial e alterações fisiológicas ${ }^{8,20,21}$. A pontuação total varia de zero a 21 pontos em recémnascidos prematuros e indicam dor acima de seis pontos. Em geral as escalas são pouco específicas para os muito prematuros, nascidos com menos de 27 semanas. A PIPP foi desenvolvida considerando essa faixa etária, pois inclui idade gestacional na avaliação ${ }^{22}$.

Recém-nascidos prematuros, com idades gestacionais menores, respondem aos estímulos dolorosos de maneira aguda e com respostas fisiológicas diferentes daqueles com idade gestacional maiores ${ }^{22}$. As escalas com base na observação desses comportamentos apresentam fragilidade na avaliação da dor sentida nessa população 23 . Em revisão sistemática para avaliar a confiabilidade, validação, viabilidade e utilidade clínica da PIPP, evidenciou-se que esta escala 
é uma medida confiável para avaliar a dor relacionada a procedimentos em recém-nascidos ${ }^{24}$.

O controle da dor em recém-nascidos prematuros melhora sua estabilidade clínica e diminui complicações ${ }^{15}$. Há evidências que a intervenção pode ser benéfica para pre-termos, atenuando as respostas frente à dor relacionada aos procedimentos ${ }^{25}$. A contenção nos neonatos realizada por meio de cueiros ou das mãos apresenta benefícios de baixo custo e efeito sobre o tempo de choro e qualidade de sono. Pois a manutenção da postura em flexão auxilia na autorregulação $\frac{26}{}$. A contenção proporcionou estabilidade clínica e redução dos escores de dor em RNPT27. A contenção facilitada inibe as respostas relacionadas ao estresse e as alterações fisiológicas, reforçando a estabilização comportamental, podendo ser usado em procedimentos de rotina nos cuidados intensivos ${ }^{28}$. Este resultado concorda com outro estudo transversal controlado e randomizado em recémnascidos prematuros avaliando o efeito da contenção facilitada, e houve diminuição dos escores de dor durante a aspiração endotraqueal 29 . Evidenciando que a contenção é benéfica, diminuindo a presença de dor no grupo estudado, podendo ser pela inibição da movimentação ativa dos recém-nascidos contidos, promovendo conforto e estabilidade motora.

Em um panorama mundial, no Japão, um estudo mostrou que a contenção realizada no momento doloroso atua como fator protetor do sistema nervoso neonatal: o toque terapêutico modificou as alterações de oxigenação cerebral, evidenciando que o toque/contenção tem efeito protetor sobre a auto regulação do fluxo sanguíneo cerebral durante a exposição de dor em recém-nascidos ${ }^{30}$. Na Malásia, outro estudo avaliou a contenção com cueiros em RNPT como alternativa à sucção, evitando assim o uso de chupetas na referida unidade. Os resultados deste estudo também evidenciam que a contenção reduziu a dor nos neonatos estudados ${ }^{31}$. As combinações não farmacológicas durante os procedimentos dolorosos facilitam a organização motora e o sono dos recém-nascidos ${ }^{28}$.

Os RNPT avaliados mantiveram os parâmetros fisiológicos observados dentro dos limites da normalidade. Destacando-se a frequência cardíaca que apresentou uma variação menor no grupo Contenção. Sendo este um resultado que evidencia que o posicionamento em contenção dos neonatos promove estabilidade clínica. Sabe-se que as alterações de frequência respiratória e frequência cardíaca não são específicas para avaliar dor e não podem ser utilizadas isoladamente ${ }^{32}$. E que frente à dor se comportam com elevação da frequencia cardíaca e respiratória e com diminuição da saturação de oxigênio.

Concordando com este estudo, outra investigação em unidade de cuidados terciários, avaliou as respostas fisiológicas frente à punção de calcâneo comparando a sucção não nutritiva com contenção em recém-nascidos prematuros. Não observaram diferenças entre os grupos de estímulo doloroso e não doloroso 28 .

Esta pesquisa proporcionou à unidade estudada, por meio da capacitação da equipe, um momento de reflexão a respeito da dor neonatal. Oferecendo educação permanente a toda equipe multiprofissional, objetivando a avaliação sistemática da dor, propondo previamente ao evento doloroso medidas não farmacológicas para prevenção e alívio, com a estruturação de protocolo de medidas não farmacológicas direcionadas aos prematuros de acordo com a idade gestacional e evento doloroso que serão submetidos. Possibilitando assim uma padronização nas estratégias de cuidado, sabendo-se que a contenção se mostrou benéfica durante a aspiração. Proporcionando aos RNPT redução da dor e desconforto, minimizando os danos decorrentes deste procedimento, repercutindo diretamente na melhora da qualidade de vida pós-internamento.

\section{Considerações finais}

Como limitações deste estudo temos a falta de controle sobre os demais eventos dolorosos que o recém-nascido recebia, pois dependiam da necessidade clínica. Porém, os horários da coleta da pesquisa ocorreram sempre depois de um período onde o RN não recebia outros procedimentos e manuseios pela equipe. A aplicação das escalas de dor foi dependente do grupo avaliador, sem padrão ouro disponível na literatura para comparação.

A intervenção não farmacológica de contenção no momento da aspiração de secreções de vias aéreas proporciona alívio da dor quando estes dados são avaliados de modo dependente. Pois os indivíduos passaram a não pontuar escores positivos para dor quando foram contidos. A intervenção de contenção por meio de cueiros mostra interferência na frequência cardíaca, onde os pacientes do Grupo Contenção 
apresentaram menor variação quando comparados ao Grupo Ninho. As demais variáveis fisiológicas avaliadas não apresentaram variação com significância estatística. Como ambas as intervenções são posturais, pode ser justificativa para não haver diferenças significativas entre os resultados obtidos.

\section{Agradecimentos}

Agradecemos a toda equipe da UTI Neonatal, em especial ao grupo de enfermeiras e fisioterapeutas que participaram dos treinamentos e mostraram a preocupação com o tema estudado, comprometendo-se com a pesquisa.

\section{Contribuições das autoras}

Andreazza MG participou da concepção, delineamento, coleta de dados, busca e análise estatística dos dados da pesquisa, interpretação dos resultados e redação do artigo científico. Motter AA participou do delineamento, interpretação dos resultados e redação do artigo. Cat MNL participou do delineamento, análise estatística e interpretação dos resultados. Silva RPGVC participou da concepção e delineamento, orientação e revisão do artigo.

\section{Conflitos de interesses}

Nenhum conflito financeiro, legal ou político envolvendo terceiros (governo, empresas e fundações privadas, etc.) foi declarado para nenhum aspecto do trabalho submetido (incluindo, mas não se limitando a subvenções e financiamentos, participação em conselho consultivo, desenho de estudo, preparação de manuscrito, análise estatística, etc.).

\section{Referências}

1. Barbosa AL, Cardoso MVLML, Brasil TB, Sochi CGS. Aspiração do tubo orotraqueal e de vias aéreas superiores : alterações nos parâmetros fisiológicos em recém-nascidos. Rev LatinoAm Enferm. 2011;19(6): 1369-1376. doi: 10.1590/S010411692011000600013

2. Roofthooft DWE, Simons SHP, Anand KJS, Tibboel D, Van Dijk M. Eight years later, are we still hurting newborn infants? Neonatology. 2014;105(3):218-26. doi: 10.1159/000357207

3. Prestes ACY, Balda RCX, Santos GMS, Rugolo LMSS, Bentlin MR, Magalhães $M$ et al. Painful procedures and analgesia in the NICU : what has changed in the medical perception and practice in a ten-year period ? J Pediatr. 2016;92(1):88-95. doi: 10.1016/j. jped.2015.04.009
4. Golianu B, Krane E, Seybold J, Almgren C, Anand KJS. Non-pharmacological techniques for pain management in neonates. Semin Perinatol. 2007;31(5):318-22. doi: 10.1053/j. semperi.2007.07.007

5. Cignacco E, Hamers J, Van Lingen RA, Stoffel L, Büchi S, Müller R et al. Neonatal procedural pain exposure and pain management in ventilated preterm infants during the first 14 days of life. Swiss Med Wkly. 2009;139(15-16):226-32.

6. Grunau RE. Neonatal pain in very preterm infants: long-term effects on brain, neurodevelopment and pain reactivity. Rambam Maimonides Med J. 2013;4(4):e0025. doi: 10.5041/RMMJ.10132

7. Motta GCP. Adaptação Transcultural E Validação Clínica Da Neonatal Infant Pain Scale Para Uso No Brasil [dissertação]. Universidade Federal do Rio Grande do Sul; 2013.

8. Bueno M, Costa P, Oliveira AAS, Cardoso R, Kimura AF. Tradução e adaptação do premature infant pain profile para a língua Portuguesa. Texto e Contexto Enferm. 2013;22(1):29-35.

9. Andreazza MG, Motter AA, Cat ML, Silva RPGVC. Percepção da dor em neonatos pela equipe de enfermagem de unidade de terapia intensiva neonatal. Rev Bras Pesq Saúde. 2017;19(4):133-9.

10. Van Marter LJ, Allred EN, Pagano M, Sanocka U, Parad R, Moore $\mathrm{M}$ et al. Do Clinical Markers of Barotrauma and Oxygen Toxicity Explain Interhospital Variation in Rates of Chronic Lung Disease? Pediatrics. 2000;105(6):1194-201. doi: $10.1542 /$ peds.105.6.1194

11. Johnston C, Zanetti NM, Comaru T, Ribeiro SNS, Andrade LB, Santos SLL. I Brazilian guidelines for respiratory physiotherapy in pediatric and neonatal intensive care units. Rev Bras Ter Intensiva. 2012;24(2):119-29. doi: 10.1590/50103-507X2012000200005

12. Cone S, Pickler RH, Grap MJ, McGrath J, Wiley PM. Endotracheal suctioning in preterm infants using four-handed versus routine care. J Obstet Gynecol Neonatal Nurs. 2014;42(1):92-104. doi: 10.1111/1552-6909.12004

13. Alinejad-Naeini M, Mohagheghi P, Peyrovi $H$, Mehran A. The effect of facilitated tucking during endotracheal suctioning on procedural pain in preterm neonates: a randomized controlled crossover study. Glob J Health Sci. 2014;6(4):278-84. doi: 10.5539/ gjhs.v6n4p278

14. Cruz CT, Gomes JS, Kirchner RM, Stumm EMF. Evaluation of pain of neonates during invasive procedures in intensive care. Rev Dor. 2016;17(3):197-200. doi: 10.5935/1806-0013.20160070

15. Anand KJ. Prevention and treatment of neonatal pain. Up To Date [Internet]. 2015. Available from: http://www.uptodate.com/ contents/prevention-and-treatment-of-neonatal-pain\#H18639417 
16. Gimenez IL, Rodrigues RF, Oliveira MCF, Santos BAR, Arakaki VSNM, Santos RS et al. Temporal assessment of neonatal pain after airway aspiration. Rev Bras Ter Intensiva. 2020;32(1):66-71. doi: $10.5935 / 0103-507 x .20200011$

17. Melo GM, Lélis ALPA, Moura AF, Cardoso MVLML, Silva VM. Pain assessment scales in newborns: integrative review. Rev Paul Pediatr. 2014;32(4):395-402. doi: 10.1590/S010305822014000400017

18. Johnston CC, Stevens BJ. Experience in a neonatal intensive care unit affects pain response. Pediatrics. 1996;98(5):925-30.

19. Motta GCP, Schardosim JM, Cunha MLC. Neonatal Infant Pain Scale : Cross-Cultural Adaptation and Validation in Brazil. J Pain Symptom Manage. 2015;50(3):394-401. doi: 10.1016/j. jpainsymman.2015.03.019

20. Guinsburg R, Balda RC, Berenguel RC, Almeida MF, Tonelloto J, Santos AM et al. Behavioral pain scales assessment in neonates. J Pediatr. 1997;73:411-8. doi: 10.2223/jped.571

21. Guinsburg R. Avaliação e tratamento da dor no recém-nascido. J Pediatr. 1999;75(3):149-60.

22. Gibbins S, Stevens B, McGrath PJ, Yamada J, Beyene J, Breau $L$ et al. Comparison of pain responses in infants of different gestational ages. Neonatology. 2007;93(1):10-8. doi: $10.1159 / 000105520$

23. Holsti L, Grunau RE, Shany E. Assessing pain in preterm infants in the neonatal intensive care unit: moving to a "brain-oriented" approach. Pain Manag. 2011;1(2):171-9. doi: 10.2217/pmt.10.19

24. Stevens B, Johnston C, Taddio A, Gibbins S, Yamada J. The premature infant pain profile: evaluation 13 years after development. Clin J Pain. 2010;26(9):813-30. doi: $\underline{10.1097 /}$ AJP.0b013e3181ed1070
25. Obeidat H, Kahalaf I, Callister LC, Froelicher ES. Use of Facilitated Tucking for Nonpharmacological Pain Management in Preterm Infants. J Perinat Neonatal Nurs. 2009;23(4):372-7. doi: 10.1097/JPN.0b013e3181bdcf77

26. Sleuwen BE, Engelberts AC, Boere-Boonekamp MM, Kuis W, Schulpen TWJ, L'Hoir MP. Swaddling: a systematic review. Pediatrics. 2007;120(4):e1097-106. doi: 10.1542/peds.2006-2083

27. Nicolau CM, Pigo JDC, Bueno M, Falcão MC. Avaliação da dor em recém-nascidos prematuros durante a fisioterapia respiratória. Rev Bras Saúde Mater Infant. 2008;8(3):285-90. doi: $\underline{10.1590 / \text { S1519-38292008000300007 }}$

28. Liaw JJ, Yang L, Wang KWK, Chen CM, Chang YC, Yin T. Nonnutritive sucking and facilitated tucking relieve preterm infant pain during heel-stick procedures: A prospective, randomised controlled crossover trial. Int J Nurs Stud. 2012;49(3):300-9.

29. Peyrovi H, Alinejad-Naeini M, Mohagheghi P, Mehran A. The effect of facilitated tucking position during endotracheal suctioning on physiological responses and coping with stress in premature infants: a randomized controlled crossover study. J Matern Fetal Neonatal Med. 2014;27(15):1555-9. doi: $\underline{10.3109 / 14767058.2013 .868429}$

30. Honda N, Ohgi S, Wada N, Loo KK, Higashimoto Y, Fukuda K. Effect of therapeutic touch on brain activation of preterm infants in response to sensory punctate stimulus: a near-infrared spectroscopy-based study. Arch Dis Child Fetal Neonatal Ed. 2013;98(3):244-8. doi: 10.1136/archdischild-2011-301469

31. Lopez O, Subramanian P, Rahmat N, Theam LC, Chinna K, Rosli R. The effect of facilitated tucking on procedural pain control among premature babies. J Clin Nurs. 2014;24(1-2):1-9. doi: 10.1111/jocn.12657

32. Gibbins S, Stevens B, Beyene J, Chan PC, Bagg M, Asztalos E. Pain behaviours in Extremely Low Gestational Age infants. Early Hum Dev. 2008;84(7):451-8. doi: 10.1016/j. earlhumdev.2007.12.007 